\title{
Analysis of galanin receptor GALR2 in multiple sclerosis
}

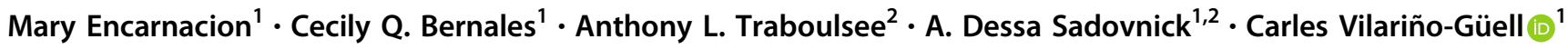

Received: 28 September 2018 / Revised: 9 September 2019 / Accepted: 2 October 2019 / Published online: 14 October 2019

(c) Springer Nature Limited 2019

Over the last few years, the implementation of whole exome sequencing (WES) technologies has nominated several pathogenic mutations for familial forms of multiple sclerosis (MS) [1-3]. Recently, Garcia-Rosa et al. described a homozygous loss-of-function mutation in galanin receptor 2 (GALR2 p.Trp249Leu, rs61745847) in a female patient who developed MS at 22 years of age [4]. The validity of WES studies, and the existence of pathogenic mutations for Mendelian forms of MS, has proven a controversial topic $[5,6]$. Therefore, it is important to assess newly identified mutations in additional populations of MS patients and healthy controls, to confirm their role in the pathophysiology of disease.

To further define the contribution of GALR2 p.Trp249Leu in the onset of MS, we genotyped rs61745847 in 2433 patients diagnosed with MS and 1066 healthy controls. All participants reported European ancestry, and were collected through the longitudinal Canadian Collaborative Project on Genetic Susceptibility to Multiple Sclerosis. Basic demographics for this population have been provided elsewhere $[7,8]$. The ethical review board at the University of British Columbia approved the study, and all participants provided informed consent. Genotyping rs61745847 in these MS patients and controls using a Taqman probe, did not identify any GALR2 p.Trp249Leu homozygote carriers. However, $46 \mathrm{MS}$ patients and 21 healthy controls were found to carry one copy of the mutation, resulting in a minor allele frequency of 0.94 and $0.98 \%$, respectively (Table 1), suggesting that heterozygote mutation carriers are not at an increased risk of developing MS (Fisher's $p=0.89$ ).

Carles Vilariño-Güell

carles@can.ubc.ca

1 Department of Medical Genetics, University of British Columbia, Vancouver, BC, Canada

2 Division of Neurology, Faculty of Medicine, University of British Columbia, Vancouver, BC, Canada
To investigate whether other potentially pathogenic GALR2 mutations are present in the Canadian MS cohort, we mined WES data from 483 MS patients for homozygote or compound heterozygote mutations, resulting in missense or nonsense GALR2 substitutions. This genetic resource was generated using an Ion Ampliseq exome kit and an Ion Torrent Proton sequencer with $100 \times$ minimum average sequencing depth, as previously described [7]. Mining WES data from MS patients did not identify potentially pathogenic mutations in GALR2. One patient carrying a heterozygous p.Pro130Ala (rs761610755) variant, and five p.Ser346Arg (rs8192514) heterozygote carriers were identified. The prevalence of these mutations in our cohort is consistent with the allelic frequencies reported in The Genome Aggregation Database for samples of European ancestry (0.003 and $0.58 \%$ ) [9], indicating that they are unlikely to play a role in the onset of MS.

In summary, the genetic characterization of GALR2 in MS patients and controls of European ancestry from Canada did not provide additional support for this galanin receptor subtype having a role in the biological processes leading to the development of disease. Given the rs61745847 minor allele frequency observed in our study, we can extrapolate that 3300 Canadians are homozygote for GALR2 p. Trp249Leu, assuming a Canadian population of 36 million and a homozygote frequency of $0.0096^{2}$. If this mutation was sufficient for the development of clinical MS symptoms, GALR2 would account for $\sim 3.3 \%$ of the $\sim 100,000$ Canadians diagnosed with MS [10], thus resulting in numerous homozygote carriers in our cohort. The lack of such homozygote patients suggests that GALR2 p.Trp249Leu may only have a role in the pathophysiology of MS, if additional yet to be determined genetic and/or environmental factors are also present. Alternatively, despite the known GALR2 role in neurogenesis and neuronal healthy, and the observed cellular miss localization of GALR2 p.Trp249Leu [4]; it is possible that one of the other four rare homozygote and predicted deleterious mutations identified by Garcia-Rosa et al., or a mutation overlooked in their analysis, is responsible for the onset of MS in their 
Table 1 Sample descriptives, and genotype frequency and statistical analysis for GALR2 rs61745847 in MS patients and controls

\begin{tabular}{llllllll}
\hline & M:F ratio & Age & AAO & \multicolumn{2}{l}{ rs61745847 } & & Fisher's \\
& & Mean \pm SD & Mean \pm SD & GG & GT & MAF $(\%)$ & $p$ value \\
\hline Controls & $1: 0.96$ & $30.8 \pm 9.6$ & NA & 1045 & 21 & 0.98 & 0.89 \\
Patients & $1: 2.78$ & $46.6 \pm 11.7$ & $67.2 \pm 10.0$ & 2387 & 46 & 0.94 & \\
\hline
\end{tabular}

M:F male:female; $A A O$ age at onset of MS, $S D$ standard deviation, $M A F$ minor allele frequency, NA not applicable patient. Thus, further genetic analysis characterizing GALR2 in MS patients, and in particular the p.Trp249Leu (rs61745847) mutation, are warranted to confirm a role for galanin receptors in the pathological mechanisms leading to the onset of MS.

Acknowledgements This research was funded by the Michael Smith Foundation for Health Research (16827), Canadian Institutes of Health Research (MOP-137051), and the Vancouver Foundation (ADV14-1597).

\section{Compliance with ethical standards}

Conflict of interest The authors declare that they have no conflict of interest.

Publisher's note Springer Nature remains neutral with regard to jurisdictional claims in published maps and institutional affiliations.

\section{References}

1. Wang Z, Sadovnick AD, Traboulsee AL, Ross JP, Bernales CQ, Encarnacion M, et al. Nuclear receptor NR1H3 in familial multiple sclerosis. Neuron. 2016;90:948-54.

2. Sadovnick AD, Gu BJ, Traboulsee AL, Bernales CQ, Encarnacion $\mathrm{M}$, Yee IM, et al. Purinergic receptors P2RX4 and P2RX7 in familial multiple sclerosis. Hum Mutat. 2017;38:736-44.
3. Vilarino-Guell C, Zimprich A, Martinelli-Boneschi F, Herculano B, Wang Z, Matesanz F, et al. Exome sequencing in multiple sclerosis families identifies 12 candidate genes and nominates biological pathways for the genesis of disease. PLoS Genet. 2019; 15:e1008180.

4. Garcia-Rosa S, Trivella DB, Marques VD, Serafim RB, Pereira JG, Lorenzi JC, et al. A non-functional galanin receptor-2 in a multiple sclerosis patient. Pharmacogenomics J. 2018; 19:72-82.

5. Wang Z, Sadovnick AD, Traboulsee AL, Ross JP, Bernales CQ, Encarnacion M, et al. Case-control studies are not familial studies. Neuron. 2016;92:339-41.

6. International Multiple Sclerosis Genetics Consortium. NR1H3 p. Arg415Gln is not associated to multiple sclerosis risk. Neuron. 2016;92(Oct):333-5.

7. Traboulsee AL, Sadovnick AD, Encarnacion M, Bernales CQ, Yee IM, Criscuoli MG, et al. Common genetic etiology between "multiple sclerosis-like" single-gene disorders and familial multiple sclerosis. Hum Genet. 2017;136:705-14.

8. Traboulsee AL, Bernales CQ, Ross JP, Lee JD, Sadovnick AD, Vilarino-Guell C. Genetic variants in IL2RA and IL7R affect multiple sclerosis disease risk and progression. Neurogenetics. 2014;15:165-9.

9. Lek M, Karczewski KJ, Minikel EV, Samocha KE, Banks E, Fennell T, et al. Analysis of protein-coding genetic variation in 60,706 humans. Nature. 2016;536:285-91.

10. Amankwah N, Marrie RA, Bancej C, Garner R, Manuel DG, Wall $\mathrm{R}$, et al. Multiple sclerosis in Canada 2011 to 2031: results of a microsimulation modelling study of epidemiological and economic impacts. Health Promot Chronic Dis Prev Can. 2017;37: $37-48$. 